\title{
Matrix-Assisted Laser Desorption Ionization Mapping of Lysophosphatidic Acid Changes after Traumatic Brain Injury and the Relationship to Cellular Pathology
}

Whitney S. McDonald, ${ }^{*}$ Elizabeth E. Jones, ${ }^{\dagger}$ Jonathan M. Wojciak, ${ }^{\ddagger}$ Richard R. Drake, ${ }^{\dagger}$ Roger A. Sabbadini, ${ }^{\ddagger}$ and Neil G. Harris ${ }^{\star}$

From the UCLA Brain Injury Research Center, * Department of Neurosurgery, David Geffen School of Medicine, University of California, Los Angeles, Los Angeles, California; the Medical University of South Carolina Proteomics Center, ${ }^{\dagger}$ Charleston, South Carolina; and Lpath, Inc., ${ }^{\ddagger}$ San Diego, California

\author{
Accepted for publication \\ May 16, 2018. \\ Address correspondence to \\ Neil G. Harris, Ph.D., Depart- \\ ment of Neurosurgery, David \\ Geffen School of Medicine at \\ UCLA, 300 Stein Plaza, Ste \\ 535, Box 956901, Los Angeles, \\ CA 90095-7039. E-mail: \\ ngharris@ucla.edu.
}

\begin{abstract}
Lysophosphatidic acid (LPA) levels increase in the cerebrospinal fluid and blood within 24 hours after traumatic brain injury (TBI), indicating it may be a biomarker for subsequent cellular pathology. However, no data exist that document this association after TBI. We, therefore, acquired matrixassisted laser desorption ionization imaging mass spectrometry data of LPA, major LPA metabolites, and hemoglobin from adult rat brains at 1 and 3 hours after controlled cortical impact injury. Data were semiquantitatively assessed by signal intensity analysis normalized to naïve rat brains acquired concurrently. Gray and white matter pathology was assessed on adjacent sections using immunohistochemistry for cell death, axonal injury, and intracellular LPA, to determine the spatiotemporal patterning of LPA corresponding to pathology. The results revealed significant increases in LPA and LPA precursors at 1 hour after injury and robust enhancement in LPA diffusively throughout the brain at 3 hours after injury. Voxel-wise analysis of LPA by matrix-assisted laser desorption ionization and $\beta$-amyloid precursor protein by immunohistochemistry in adjacent sections showed significant association, raising the possibility that LPA is linked to secondary axonal injury. Total LPA and metabolites were also present in remotely injured areas, including cerebellum and brain stem, and in particular thalamus, where intracellular LPA is associated with cell death. LPA may be a useful biomarker of cellular pathology after TBI. (Am J Pathol 2018, 188: 1779-1793; https://doi.org/10.1016/ j.ajpath.2018.05.005)
\end{abstract}

Traumatic brain injury (TBI) affects nearly 1.7 million individuals each year, many of who sustain functional impairments from progressive axonal injury and neuronal death after trauma. Because of the complex pathology of TBI, there is no effective treatment to restore function in patients. The rapid onset of pathology and the complexity of the cellular response to TBI suggest that there are early signaling molecules involved in initiating the cascade of cellular events that promote functional impairment after trauma. $^{1-4}$ Lysophosphatidic acid (LPA) is one such molecule shown to be significantly involved in the pathology of central nervous system (CNS) injury. ${ }^{5-7}$
LPA and its metabolites act as potent cellular messengers $^{8}$ and are likely to be involved in much of the early pathologic processes after TBI, as indicated by overlapping data on the inflammatory cascade, ${ }^{9}$ altered calcium homeostasis, ${ }^{10,11}$ astrogliosis, ${ }^{12,13}$ neurite retraction, ${ }^{14,15}$ and cell death. ${ }^{16}$ The parallel between the cellular

Supported by NIH grants R44 NS87641 (R.A.S., N.G.H.) and R01 NS091222 (N.G.H.) and the UCLA Brain Injury Research Center.

Disclosures: Human anti-lysophosphatidic acid antibodies were supplied by Lpath, Inc. (San Diego, CA). J.M.W. and R.A.S. are employees of Lpath, Inc. 
responses to LPA and trauma-induced cellular pathologies suggests that LPA may be a useful biomarker for TBI. This is supported by a recent study demonstrating that LPA is significantly increased in the cerebrospinal fluid (CSF) 3 hours after injury in a mouse model of TBI as well as within 24 to 36 hours after injury in a subset of patients with severe TBI. $^{5}$ The CSF concentration of phospholipids related to LPA has also been shown to be significantly higher in patients with poor outcomes. ${ }^{17}$ Activation of LPA receptor type 1 has been shown to contribute to the pathology of spinal cord injury. ${ }^{18}$ Attenuating LPA signaling with anti-LPA antibodies has proved successful in improving recovery after a rodent model of $\mathrm{TBI}^{5}$ and spinal cord injury, ${ }^{6}$ suggesting that enhanced LPA production is involved in injury pathology after CNS trauma.

A comprehensive understanding of the contribution that LPA makes to TBI pathology is required to successfully address aberrant LPA signaling, including the source(s) of LPA changes, the spatial extent of LPA expression, and the duration in which aberrant LPA levels are present in the injured brain. Furthermore, it is also important to determine the effect of injury on accumulation of LPA and its metabolites in brain tissue, because LPA alone can initiate the production of more LPA within adjacent cells, ${ }^{19,20}$ generating a feed-forward mechanism of LPA expression and signaling in the injured brain. Despite this knowledge, no study has determined if the levels of LPA actually change within the injury epicenter and regions distal to the injury site, and whether this change is associated with known markers of pathology. LPA and its associated phospholipids are essential for LPA metabolic regulation, cellular function, and signaling; thus, identifying the spatial-temporal disruption of LPA metabolic changes will be a crucial step in understanding the role of LPA in the pathogenesis of TBI. The results herein are to identify spatiotemporal LPA dysregulation within 3 hours of TBI in the rat brain.

Matrix-assisted laser desorption ionization imaging mass spectrometry (MALDI IMS) is a sensitive label-free approach capable of producing ion-density maps representing the distribution of a variety of analyses linked directly with tissue histopathology. ${ }^{21-26}$ Using highresolution MALDI IMS and immunohistochemistry (IHC), we determined whether changes in lipid distribution after controlled cortical impact (CCI) model correlate with markers of TBI pathology, such as axonal injury and necrosis. Brain tissue slices obtained from control and CCI rats at 1 and 3 hours after injury were analyzed via MALDI IMS. Modulation in the distribution of LPA was observed not only at the site of injury, but also in distal regions, suggesting that activated platelets within hemorrhagic areas of the brain are not the only source of enhanced LPA after injury. Using IHC, it was confirmed that increases of LPA in gray and white matter regions were closely associated with markers of neural degeneration and axonal damage, suggesting that early phospholipid changes may be useful biomarkers of pathology and longer-term functional impairments associated with axonal injury and cell death.

\section{Materials and Methods}

\section{Experimental Protocol}

Fresh frozen rat brains were collected from control animals or those undergoing injury, collected at 1 and 3 hours after CCI brain injury. Frozen brains $(n=3$ per group) were sectioned and analyzed using MALDI IMS to visualize lipid distribution across the brains. Adjacent coronal sections were also collected for IHC.

\section{Animals}

Adult (3-month-old) male Sprague-Dawley rats (220 to $250 \mathrm{~g}$ ) were purchased from Charles River Laboratories (Hollister, CA). All experiments were conducted in accordance with the University of California, Los Angeles, Chancellor's Animal Research Committee and the Public Health Service Policy on Humane Care and Use of Laboratory Animals.

\section{Injury}

All rats were anesthetized with $3 \%$ to $4 \%$ isoflurane (2\% maintenance) vaporized in oxygen flowing at $0.8 \mathrm{~L} /$ minute. After hair removal from the head and scalp and cleaning with betadine scrubs, the animal was positioned within a stereotaxic frame (Stoelting Co, Wood Dale, IL). A longitudinal skin incision was made, followed by a 6-mm-diameter craniotomy centered at $0.5 \mathrm{~mm}$ posterior to bregma and $1 \mathrm{~mm}$ lateral to the midline using a dental drill. Body temperature was maintained throughout the surgery using a thermostatically controlled heating pad (Harvard Apparatus, Holliston, MA). Cortical injury was performed with a flat 3$\mathrm{mm}$-diameter metal tip attached to an impactor rod and launched onto the dura using $20 \psi$, and to a depth of $2 \mathrm{~mm}$ below the dura. The craniotomy was covered with a layer of nonbioreactive silicone elastomer (Kwik-Cast, Sarasota, FL) before suturing the wound closed.

\section{MALDI IMS}

\section{Spectra Acquisition}

After terminal pentobarbital anesthesia $(50 \mathrm{mg} / \mathrm{kg})$, rats were perfused with phosphate-buffered saline $(0.1 \mathrm{~mol} / \mathrm{L})$ via transcardial perfusion to remove blood. Brains from injured and sham rats were collected at 1 and 3 hours after injury ( $n=3$ per group). Tissues were extracted and snap frozen under 2-methylbutane at $-30^{\circ} \mathrm{C}$, sectioned at $12 \mu \mathrm{m}$, and thaw mounted onto indium tin oxide-coated slides (Bruker Daltonics, Billerica, MA). Representative brain sections from all three groups $(n=3$ sections per brain) 
were matched to the same anteroposterior plane close to the level of the contusion core (bregma, $-3 \mathrm{~mm}$ ). Lipids were detected in positive ion mode on a Bruker Daltonics $7 \mathrm{~T}$ solariX FTICR-MALDI mass spectrometer system (Bruker Daltonics, Bremen, Germany), as previously described. ${ }^{25}$ All images were collected in the $\mathrm{m} / \mathrm{z}=150$ to 1000 range, collecting 400 shots per pixel and stepping the laser either 60 or $80 \mu \mathrm{m}$ across the tissue (as indicated per figure), using a laser spot size of $25 \mu \mathrm{m}$ in diameter. Data were collected in broadband detection mode with a trigger delay of $1 \mathrm{~mol} / \mathrm{L}$ collecting $1 \mathrm{MB}$ data per spectrum, making the achievable resolution of this data set around 150,000 for the lipid mass range of $\mathrm{m} / \mathrm{z}=400$ to 900 . Images of differentially expressed lipids were generated using FlexImaging version 4.1 software (Bruker Daltonics).

\section{Image Spectral Intensity Measurements}

Sections immediately adjacent to those used for MALDI IMS were post-fixed in $4 \%$ paraformaldehyde for $15 \mathrm{mi}-$ nutes and processed for hematoxylin and eosin stain. Whole sections were scanned to an image using a $40 \times$ objective lens and the Zeiss microscope image tiling feature of Axiovision software version 4.8.1 (Carl Zeiss Microscopy, Thornwood, NY); they were used to draw regions of interest (ROIs) with ImageJ version 1.50G (W.S. Rasband; NIH, Bethesda, MD; http://rsb.info.nih.gov/ij; last accessed September 30, 2017) within ipsilateral cortical gray matter, ipsilateral subcortical white matter (corpus callosum), and ipsilateral anterior nucleus of thalamus. ROIs from hematoxylin and eosin sections were manually scaled and aligned by superimposing them on the MALDI images in ImageJ. ROI mean spectrum intensity levels were then normalized to the corresponding uninjured control ROI values for each slide and displayed as normalized pixel-intensity units (NIUs) for each lipid detected to allow for comparison between regions and between brains. Heme spectral images of rat sections at the epicenter of the injury (bregma, -1.4) were digitally coregistered together by affine transformations, ${ }^{27}$ binarized, and then displayed as group-level overlap images to show the degree of lesion overlap between injured rats.

\section{IHC and Analysis}

Sections immediately adjacent to those used for MALDI were post-fixed in $4 \%$ paraformaldehyde for 15 minutes in two brains per group, and then processed for standard IHC using the following antibodies: rabbit anti- $\beta$-amyloid precursor protein ( $\beta$ APP; 1:100; Invitrogen; number 51-2700) and human anti-LPA (1:250; Lpath, Inc., San Diego, CA; LT3211). For $\beta A P P$ staining, antigen retrieval was performed by first boiling the sections in $10 \mathrm{mmol} / \mathrm{L}$ sodium citrate for 2 minutes and incubating the slides in the same solution for 30 minutes thereafter, after which the sections were permeabilized in $0.5 \%$ Triton $\mathrm{X}$ in tris-buffered saline for 60 minutes. After incubation with the primary antibodies, the avidin-biotin complex method was used to amplify the signal of the primary antibody for visualization with 3,3'-diaminobenzidine. Staining was processed with Fluoro-Jade B (FJ-B; 0.0004\%; Histo-Chem, Jefferson, AR). Controls (no primary antibody) were run concurrently to confirm the specificity of the antibody.

Representative ipsilateral images of FJ-B and LPA were acquired within the peri-infarct region using a $40 \times$ objective lens under fluorescence (488-nm excitation) and bright-field light, respectively, with a Zeiss M2 Axioimager microscope (Carl Zeiss Microscopy). Total numbers of FJ-B-labeled cells in the thalamus were obtained in two brains/postinjury time-point (one section/brain) with Stereoinvestigator software version 11.06.2 (MicroBrightfield, Williston, VT) and were expressed as number of cells/section. Pixel intensity maps of ipsilateral white matter $\beta$ APP were obtained using the $\mathrm{X}-\mathrm{Y}$ image tiling feature of Axiovision (Carl Zeiss Microscopy), after which the resolution was down-sampled to match the MALDI resolution using the Image feature of ImageJ. Images of $\beta A P P$ and MALDI were manually aligned using ImageJ, and the integrated pixel intensity across the entire white matter was obtained.

\section{Statistical Analysis}

Data are displayed as the means \pm SEM, and betweengroup statistical significance was tested for using $t$-test and analysis of variance with Tukey's honestly significant difference post hoc when appropriate using the R statistical package (RStudio, Inc., Boston, MA). Statistical significance $(\alpha)$ was set at $P \leq 0.05$ for all comparisons. Lipid species were treated as independent variables for statistical analysis.

\section{Results}

LPA Increases Diffusely after TBI, whereas LPA Metabolites Increase Predominantly in White Matter Regions

Although it is known that LPA accumulates within CSF after $\mathrm{TBI},{ }^{5}$ coincident with the start of the major cellularmolecular cascade leading to inflammation and cell death, ${ }^{28-30}$ it is unknown whether brain LPA concentrations increase concomitantly. To identify brain regions likely to be affected by pathologic levels of LPA after TBI, MALDI imaging and IHC were used to map brain-wide changes in extracellular and membrane-bound LPA in sagittal brain sections from the ipsilateral hemisphere harvested at 3 hours after injury and from anesthetic (uninjured) controls (Figure 1). Semiquantitative measurements of lipid changes at 3 hours after injury were normalized to control brain measurements and displayed as NIUs (Figure 1, A and B). The results show that LPA 18:1 is increased diffusely throughout the brain after injury (Figure 1, A and B) and that this is especially prevalent within the cerebellum, 
A
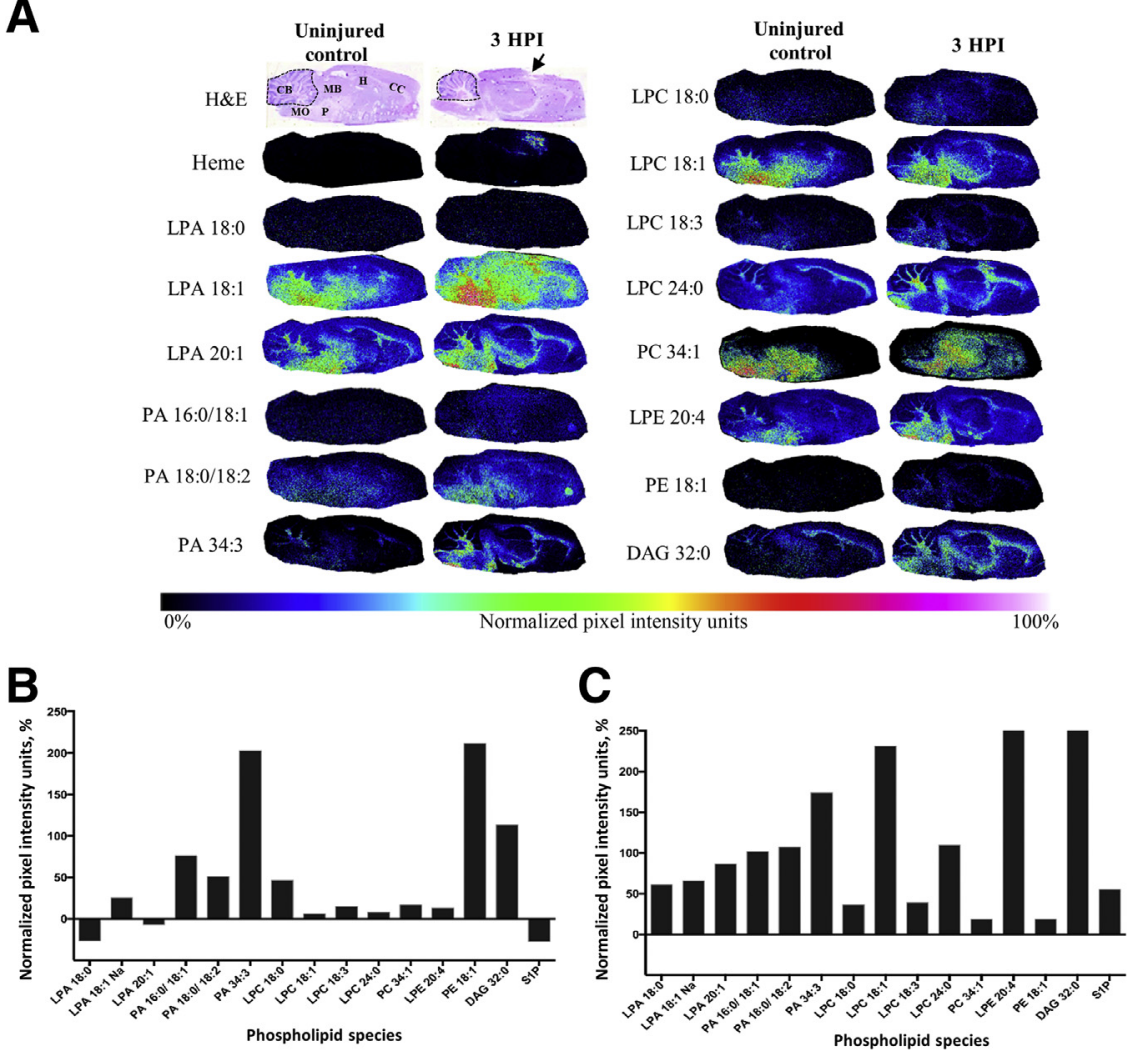

Figure 1 Brain injury enhances phospholipid metabolism in the rat brain. A: Images of sagittal brain sections of lipid species obtained by matrixassisted laser desorption ionization imaging mass spectrometry from an uninjured control and a 3 hours postinjury (HPI) rat pseudocolored by intensity to demonstrate the brain-wide change in lipid levels after injury. Images of hematoxylin and eosin (H\&E) sections from adjacent sections highlight the brain regions used for mean signal intensity analysis. Dotted lines indicate cerebellar regions of interest; arrow, the center of the injury site. B: Plot of average pixel intensity over the entire brain section for each lipid species examined for the 3 hours postinjury brain, displayed as normalized data by percentage change from uninjured control average intensity [normalized pixel-intensity units (NIUs)]. C: Plot of average pixel intensity in the cerebellum examined for the 3 hours postinjury brain; NIUs for each lipid are displayed as a percentage change from uninjured control lipid average intensity values. CB, cerebellum; CC, corpus colossus; DAG, diacylglycerol; $\mathrm{H}$, hippocampus; LPA, lysophosphatidic acid; LPC, lysophosphatidylcholine; LPE, lysophosphatidylethanolamine; $M B$, midbrain; M0, medulla oblongata; $P$, pons; $P A$, phosphatidic acid; PC, phosphatidylcholine; $P E$, phosphatidylethanolamine; S1P, sphingosine-1-phosphate. corpus callosum (CC), midbrain, medulla oblongata, and hippocampus within 3 hours after injury (Figure 1A). LPA precursors were similarly increased after injury, with a robust and widespread increase in phosphatidic acid (PA), lysophosphatidylcholine (LPC), and phosphatidylethanolamine (PE) species predominantly in white matter throughout the brain, as well as in structures remote from the site of injury (Figure 1A), including the cerebellum and its white matter tracts (Figure 1, A and B). Semiquantitative intensity measurements of the cerebellar region shown in the hematoxylin and eosin section (Figure 1A) indicate that all acyl LPA species are enhanced at least 50\% above uninjured control levels 3 hours after injury (Figure 1C). Cerebellar LPA intracellular and extracellular precursors PA and LPC were also increased from control levels, especially LPC 18:1, lysophosphatidylethanolamine $20: 4$, and diacylglycerol 32:0, which were increased from control by $>200 \%$. These observations demonstrate that acute injury profoundly alters LPA and LPA metabolite expression throughout the brain, and that this occurs especially in white matter regions at both proximal and distal sites from the injury epicenter.

\section{Total LPA and LPA Metabolites Increase within Hemorrhagic Pericontused Gray Matter Regions}

Because LPA is known to be released from activated platelets, ${ }^{31}$ it was determined if LPA was associated with parenchymal hemorrhage after TBI, in regions where the blood-brain barrier was disrupted, allowing systemic LPA sources to contaminate the brain. The CCI injury model used in this study results in increased blood-brain barrier permeability and hemorrhage at the injury epicenter (Figure 2, A-C); accordingly, elevated LPA 20:1 expression was found in these gray matter regions (Figure 2, D-L, and Figure 3, B and C; Supplemental Figures S1-S4 and Supplemental Table S1). Coregistered MALDI images of heme acquired from coronal sections through the epicenter of the injury $(-1.34 \mathrm{~mm}$ posterior from bregma) from rats at 1 and 3 hours after injury revealed a consistent heme signal present at the core injury site within the cortical gray matter (Figure 2, B and C). Semiquantitative pixel intensity measurements of heme within these regions confirmed signal increases at the epicenter compared with controls $(9.1 \pm 2.07$ and $13.4 \pm 3.74$ NIUs at 1 and 3 hours after injury, respectively; $P<0.001$ ) (Figure 3B). As expected, most of the heme was associated with the site of primary injury; however, substantial LPA and its precursors were upregulated in distal noninjured sites not associated with heme, as shown in sagittal sections (Figure 1). ROI measurements made in coronal sections, including the hemorrhagic/primary site of injury (Figure 3A), indicated significant increases in unsaturated LPA species 18:1 and 20:1 above uninjured controls in gray matter at 3 hours after injury but not earlier at 1 hour after injury $(P<0.05)$ 

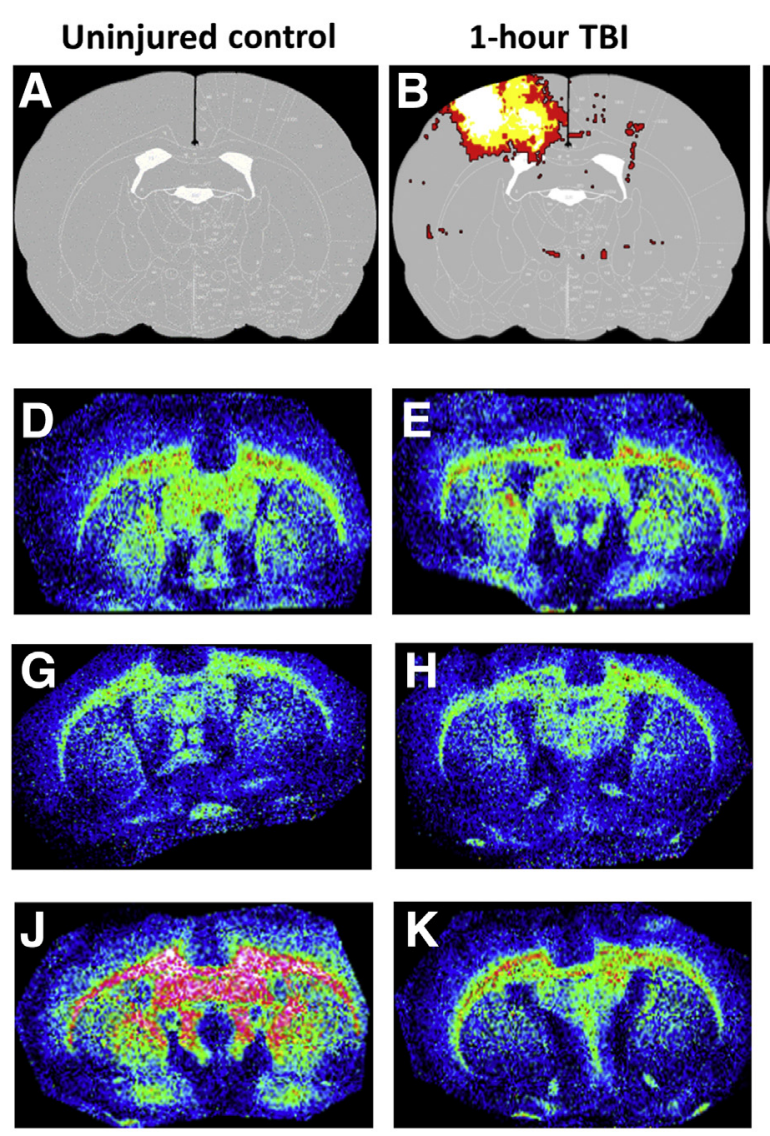

\section{3-hour TBI}
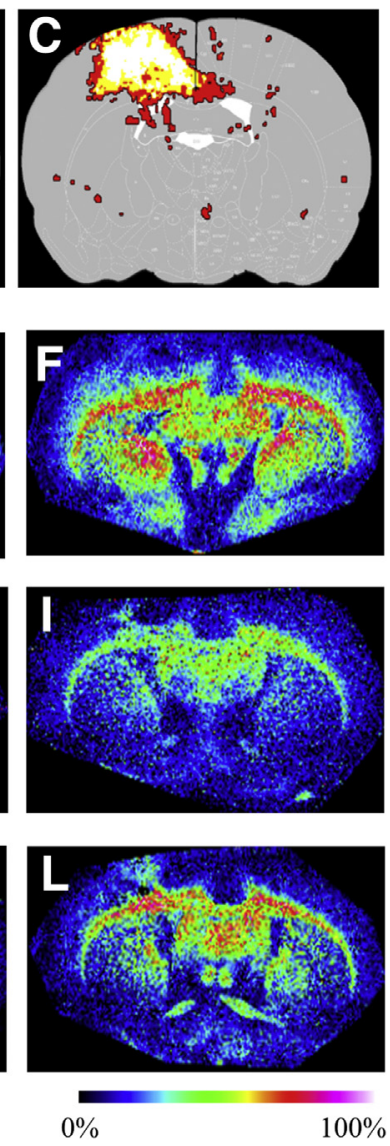

Figure 2 Overlap maps of lysophosphatidic acid (LPA)/heme signal. A-L: Calculated summary images of heme $(\mathbf{A}-\mathbf{C})$ and representative images of LPA 20:1 (D-I) from uninjured control (D, G, and $\mathbf{J}$ ) and left-hemisphere injured rats at 1 hour $(\mathbf{E}, \mathbf{H}$, and $\mathbf{K})$ and 3 hours $(\mathbf{F}, \mathbf{I}$, and $\mathbf{L})$ after injury, showing higher gray matter LPA and the lack of significant overlap of the heme signal to the increased intensity of LPA within the injury site. A-C: Heme signal images calculated from three uninjured and three injured rats at 1 and 3 hours after injury; image data overlaid on a rat atlas at $-1.3 \mathrm{~mm}$ posterior from bregma to indicate the distribution of blood at the level of the injury epicenter. Colors in $\mathbf{B}$ and $\mathbf{C}$ represent number of overlapping rat heme signals: red $=1$ rat, yellow $=2$ rats, and white $=3$ rats. Colorimetric scale indicates lipid intensity (black is lowest and white is highest presence of LPA 20:1). D-F, G-I, and $\mathbf{J}-\mathbf{L}$ : Images were obtained in three cohorts, and in each case the uninjured control was used to normalize the signal for semiquantitative analysis in gray matter in F. TBI, traumatic brain injury.
(Figure 3, A, C, and D; Supplemental Figures S1-S4 and Supplemental Table S1). However, there were no changes in unsaturated LPA species 18:0 or 20:0 at any postinjury time point. Levels of the LPA metabolites, PA 16:0/18:1 and 18:0/18:2, were greatly increased above uninjured controls by 5 to 10 NIUs at both time points after injury $(P<0.05)$ (Figure 3, A, B, and E; Table 1). Similarly, the unsaturated extracellular precursors to LPA, LPC species 18:3 and 20:1, phosphatidylcholine species 34:1, and PE 18:1, were also significantly increased at both postinjury time points by up to 5 NIUs $(P<0.05)$ (Figure 3, A, B, and F; Table 1). Although the saturated extracellular metabolic precursor of LPA, LPC 18.0, was significantly enhanced by up to 15 NIUs at 1 and 3 hours after injury, the 24:0 LPC species was significantly reduced at both times $(0.8 \pm 0.04$ and $0.8 \pm 0.04$ NIUs, at 1 and 3 hours after injury, respectively; $P<0.05$ ) (Figure $3, \mathrm{~A}$ and $\mathrm{B}$; Table 1). These data suggest that hemorrhage in the ipsilateral pericontused cortical gray matter is chiefly associated with enhanced LPA metabolism within 1 hour after injury, as well as the selective enhancement of bioactively potent LPA species $18: 1$ and $20: 1^{32}$ by 3 hours after injury.
Total LPA Decreases in Nonhemorrhagic Gray Matter, but Intracellular LPA Increases and Is Associated with Cell Death

Intracerebral hemorrhage is not the only source of enhanced LPA metabolism in the injured brain. Some cancer cells and adipocytes constitutively release LPA. ${ }^{19,33,34}$ Therefore, to investigate the postinjury changes to LPA levels in the absence of hemorrhage, MALDI IMS data were interrogated for lipid signal by comparing pixel intensities extrapolated from ROIs distal to the contusion center, but neighboring a region where cell injury or death is known to occur (Figure 4, B-M). This analysis showed that unlike the heme-associated lipid increases (Figure 3), all LPA species, as well as the precursor LPC and lysophosphatidylethanolamine, were significantly reduced at both time points after injury in the absence of heme $(P<0.05)$ (Figure 4A). Despite this, however, the LPA precursors PA 16:0/18:1, 18:0/18:2, and 34:3, PE 18:1, and LPC 18:0 and 18:3 trended toward increased production, although this was not significant $(P>0.05)$ (Figure $4 \mathrm{~A}$ and Table 1$)$. These same LPA precursors were also among the most significantly increased metabolites within the heme-containing regions 
A

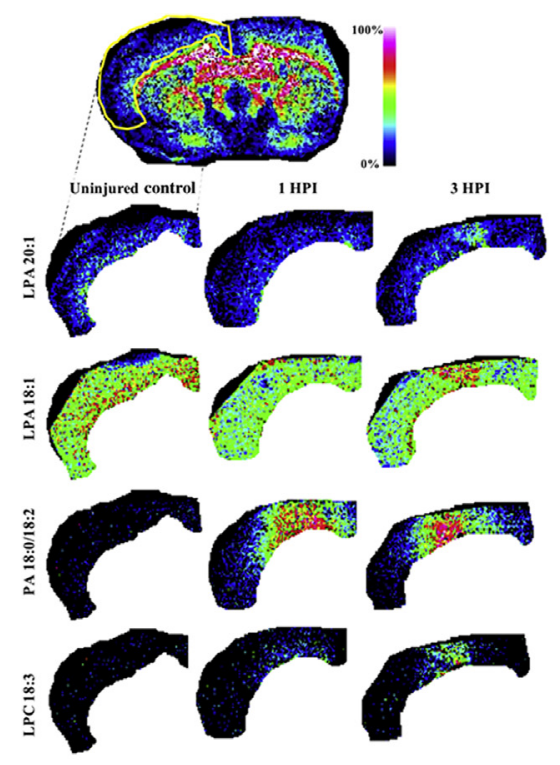

C
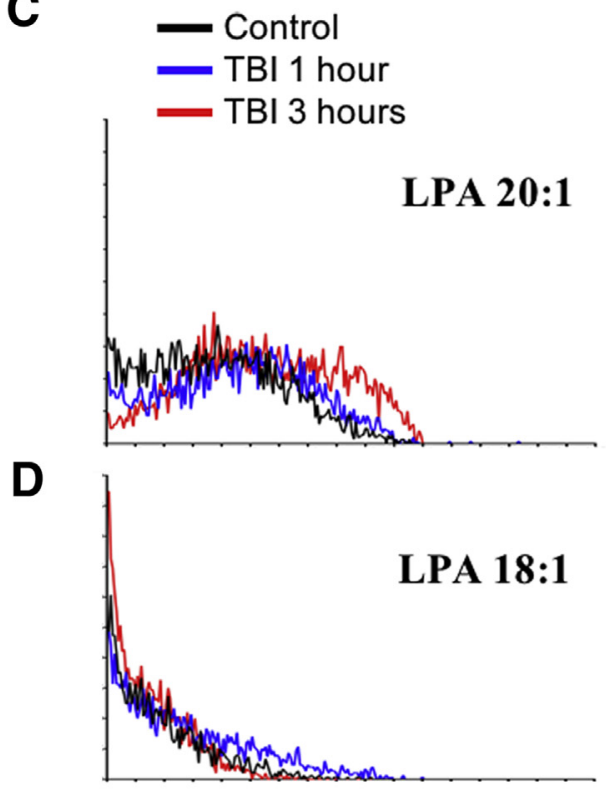

E
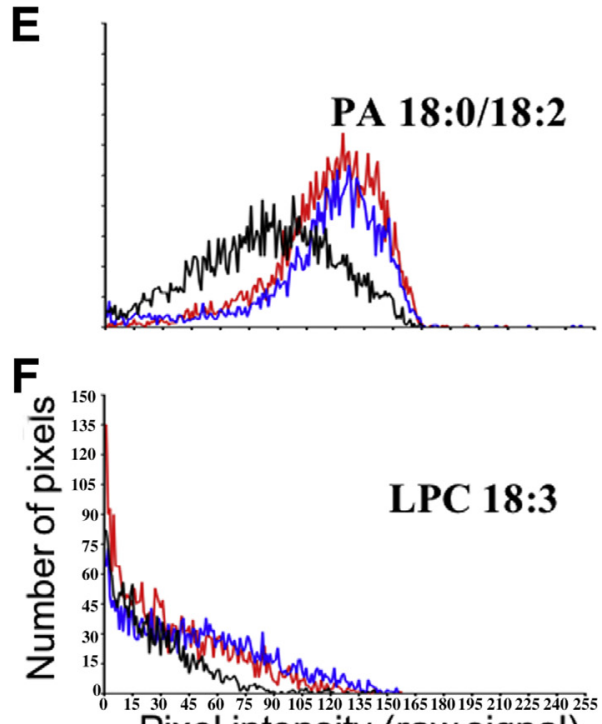

Pixel intensity (raw signal)
B

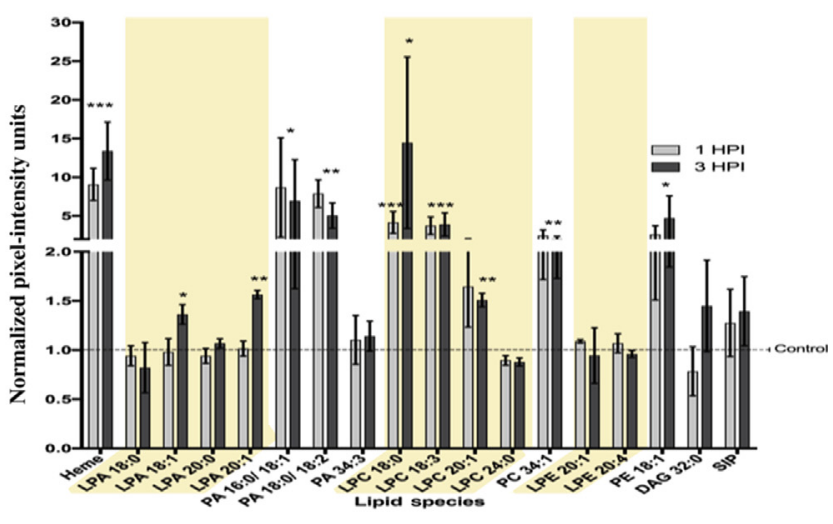

Figure 3 Lysophosphatidic acid (LPA) and LPA metabolites are increased in the ipsilateral cortical gray matter after injury in hemecontaminated regions. A: Representative matrix-assisted laser desorption ionization (MALDI) image data of cortical gray matter regions of interest (ROIs) from uninjured control and injured rats at 1 and 3 hours after injury (HPI) for different lipid species. The yellow outline in $\mathbf{A}$ represents the area shown in LPA 20:1, LPA 18:1, phosphatidic acid (PA) 18:0/18:2, and lysophosphatidylcholine (LPC) 18:3. B: Plot of all MALDI imaging mass spectrometry signal data obtained from this ROI, displayed as mean cortical gray matter signal intensity (normalized to uninjured control) at 1 and 3 hours after injury. Shaded regions highlight the 1acyl (lyso-) phospholipid species. C-F: Mean ROI pixel intensity distribution plots for LPA 20:1 (C), LPA 18:1 (D), PA 18:0/18:2 (E), and LPC 18:3 (F), indicating greater metabolite levels after injury compared with uninjured control. Data are expressed as means \pm SEM (B). $n=3$ per group ( $\mathbf{A}$ and $\mathbf{C}-\mathbf{F}) .{ }^{*} P<0.05,{ }^{* *} P<0.01$, and ${ }^{* * *} P<0.001$ for the difference between 1 and 3 hours after injury (two-tailed $t$-test, 95\% CI in R). DAG, diacylglycerol; LPE, lysophosphatidylethanolamine; PC, phosphatidylcholine; $\mathrm{PE}$, phosphatidylethanolamine; S1P, sphingosine1-phosphate; TBI, traumatic brain injury. 
Table 1 Phospholipid Species Detected in Specified Regions of Interest

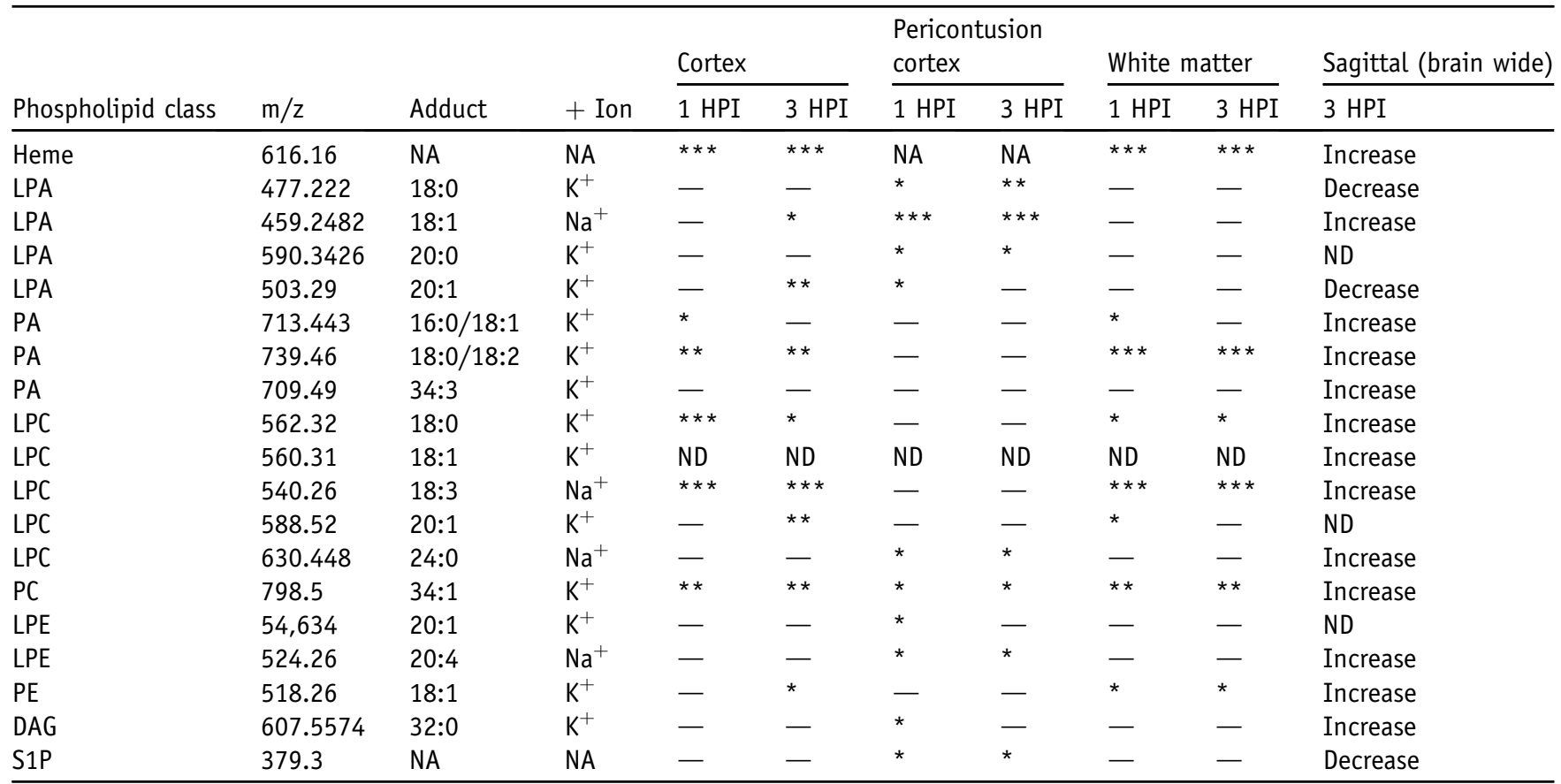

Adducts represented as carbon chain length: number of double bonds; spectra acquired in positive ion mode, potassium $\left(\mathrm{K}^{+}\right)$and sodium $\left(\mathrm{Na}^{+}\right)$. For each region of interest (ipsilateral cortex, pericontusion cortex, and ipsilateral white matter), $n=3$ per group per time period; $P$ values for pixel intensities increase (green) or decrease (red) from control value pixels and were determined by bootstrapping the $95 \%$ CIs around the median and two-way analysis of variance for each time period and each region of interest in R. Spectra not detected for a given lipid indicated the phospholipid species that were not gathered in the mass spectrometry run. Sagittal brain-wide changes are indicated by pixel intensity differences between sagittal region of interest of control and sagittal region of interest of 3 HPI ( $n=1$ per group). Brain-wide pixel intensities at 3HPI are indicated as increase or decrease from control pixel intensities. ${ }^{*} P \leq 0.05,{ }^{* *} P \leq 0.01$, and ${ }^{* * *} P \leq 0.001$.

-, Pixel intensities for a given phospholipid that did not change from control value $(P>0.05)$; DAG, diacylglycerol; heme, phospholipid class, hemeoglobin; HPI, hours after injury; LPA, lysophosphatidic acid; LPC, lysophosphatidylcholine; LPE, lysophosphatidylethanolamine; $\mathrm{m} / \mathrm{z}$, mass/charge ratio; NA, not applicable; ND, not detected; PA, phosphatidic acid; PC, phosphatidylcholine; PE, phosphatidylethanolamine; S1P, sphingosine-1-phosphate.

(Figure 3B), so that the variable increases in nonheme regions may suggest that these LPA precursors are sensitive markers of injury. The data also suggest that brain injury, in the absence of hemorrhage, is also regionally associated with modifications in LPA metabolism.

TBI pathogenesis is marked by neuronal death and altered cellular fate of neuroblasts in the brain. ${ }^{35}$ LPA can also modulate these complex cellular processes through activation of intracellular LPA receptors, whereas PA does not have this effect. ${ }^{34}$ To more accurately resolve intracellular, rather than total, LPA expression changes, brain sections adjacent to those used for MALDI IMS analysis were stained by standard IHC using a humanized antibody specific for LPA. The data revealed that, unlike the MALDI images, where a decrease in total tissue LPA within nonhemorrhagic cortical gray matter regions was found, there was a robust increase in cytoplasmic LPA within the pericontusional cortex at 3 hours after injury, compared with the same region in uninjured control tissue (Figure 4, B, F, and $\mathrm{J}$ ). To determine whether cytoplasmic enrichment of LPA is associated with markers of cell death, FJ-B is a neuron-specific stain $^{36}$ used to detect necrotic cells in adjacent sections within the same regions used for LPA detection by IHC and MALDI (Figure 4, D, E, H, I, L, and $\mathrm{M})$. The results showed a robust increase in FJ-B-positive necrotic cells in regions containing increased LPA at 3 hours after injury compared with uninjured control. Qualitatively greater FJ-B staining at 3 hours postinjury (Figure 4, J-M), compared with control, and 1 hour postinjury groups (Figure 4, B-I) was observed in regions where intracellular LPA was more readily apparent (Figure 4J compared with Figure 4F).

\section{Early Increases in LPA Occur before Cell Death in the Thalamus}

Corticothalamic atrophy is a major comorbidity of both clinical $\mathrm{TBI}^{37}$ and in the CCI model of TBI used herein. ${ }^{38}$ Because of gradual degeneration of deafferented axons, corticothalamic atrophy occurs in a relatively delayed manner compared with damage at the primary injury site. MALDI spectra from the thalamus were analyzed to determine whether early changes in LPA metabolism are temporally predictive of impending cellular damage that is known to occur in the thalamus. Thalamic ROI analysis revealed marked enhancement in LPA 18:1 expression at 

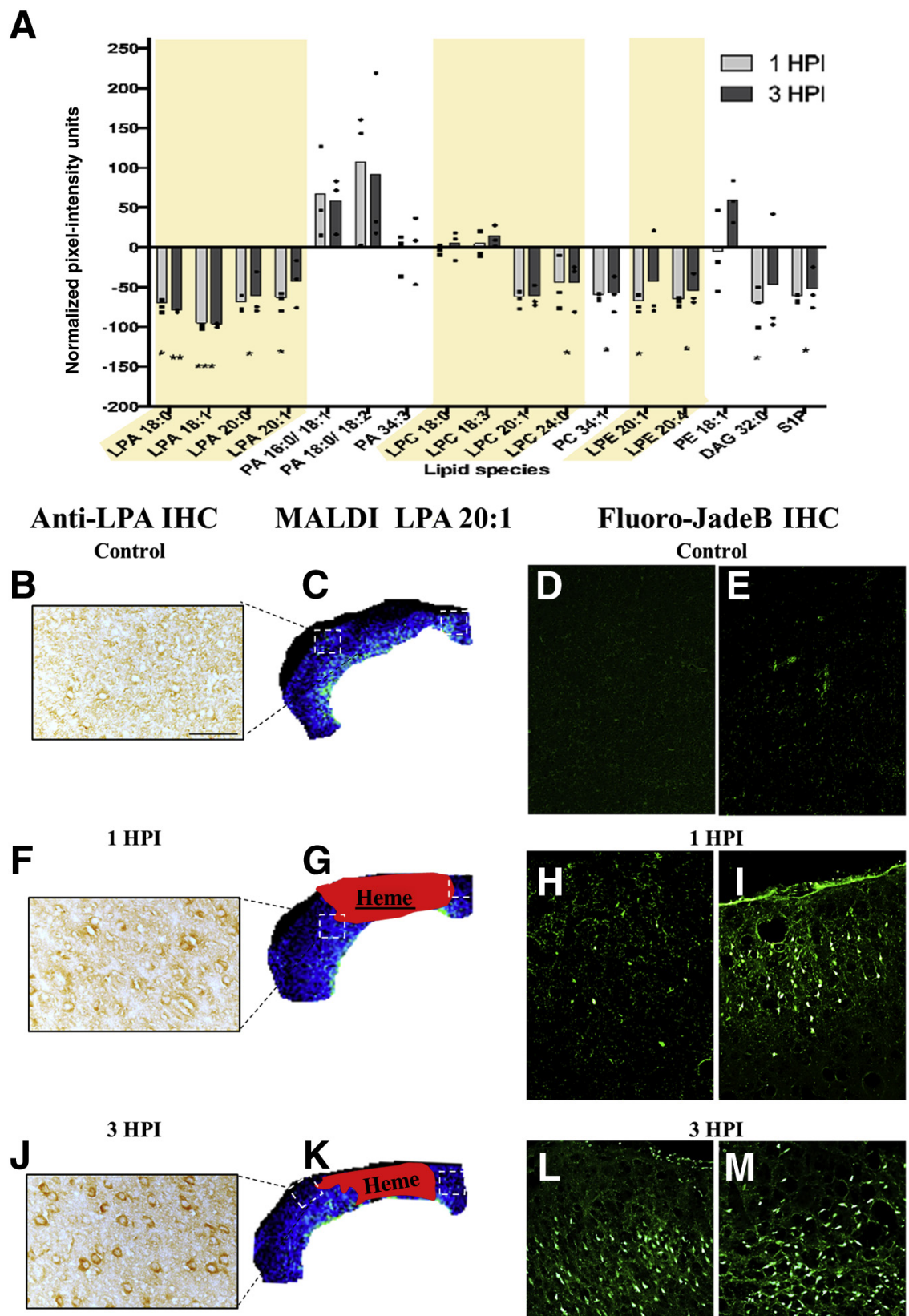

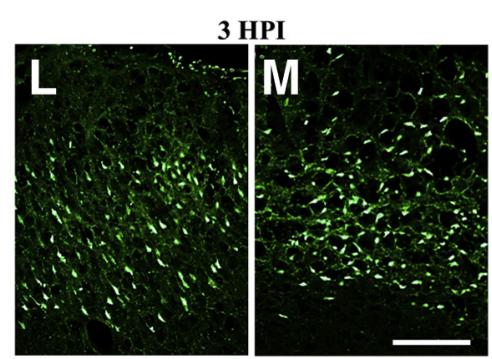

Figure 4 Matrix-assisted laser desorption ionization (MALDI)-detected lysophosphatidic acid (LPA) and LPA metabolites are decreased in the pericontusional cortex, but LPA immunohistochemistry (IHC) is increased. A: Plot of mean pericontusional cortical gray matter LPA/LPA metabolite levels at 1 and 3 hours postinjury (HPI) pixel (intensity normalized to uninjured controls); shaded regions highlight the 1-acyl (lyso-) phospholipid species. B, C, F, G, J, and K: Representative images of immunostained sections and corresponding MALDI imaging mass spectrometry data for uninjured controls (B and $\mathbf{C}$ ) and 1 ( $\mathbf{F}$ and $\mathbf{G}$ ) and 3 ( $\mathbf{J}$ and $\mathbf{K}$ ) hours after injury. $\mathbf{D}, \mathbf{E}$, H, I, L, and M: Regions of injured/necrotic cells indicated by Fluoro-Jade B immunohistochemical staining of uninjured control (D and $\mathbf{E})$ and 1 ( $\mathbf{H}$ and $\mathbf{I}$ ) and $\mathbf{3}$ ( $\mathbf{L}$ and $\mathbf{M}$ ) hours after injury, taken from the same regions of interest used for MALDI pixel intensity-based analysis for lipids in adjacent sections. Boxed areas in $\mathbf{C}, \mathbf{G}$, and $\mathbf{K}$ are shown in $\mathbf{B}, \mathbf{F}$, and $\mathbf{J}$, and also in $\mathbf{D}$ and $\mathbf{E}, \mathbf{H}$ and $\mathbf{I}$, and $\mathbf{L}$ and $\mathbf{M}$, respectively. ${ }^{*} P<0.05,{ }^{* *} P<0.01$, and $* * * P<0.001$ for the difference between 1 and 3 hours after injury (two-tailed $t$-test, $95 \% \mathrm{CI}$ in R). Scale bars: $50 \mu \mathrm{m}$ (B, F, and J); $100 \mu \mathrm{m}$ (D, E, H, I, L, and M). DAG, diacylglycerol; LPC, lysophosphatidylcholine; LPE, lysophosphatidylethanolamine; PA, phosphatidic acid; PC, phosphatidylcholine; $\mathrm{PE}$, phosphatidylethanolamine; S1P, sphingosine-1-phosphate.
1 hour after injury compared with control $(8.62 \pm 0.96$ and $1.32 \pm 0.19$ NIUs, respectively; $P<0.05$ ) (Figure 5, $\mathrm{A}$ and $\mathrm{B}$ ), and this normalized by 3 hours after injury. PA showed a similar temporal pattern of expression: increase at 1 hour after injury compared with uninjured $(3.76 \pm 1.28$ and 21 and $43.14 \pm 5.28$ NIUs, respectively; $P<0.05$ ) (Figure 5, A and B), with normalization to control levels by 3 hours after injury. Much smaller, but significant, increases in the LPA metabolite LPC 18:0 and 18:3 and PE 18:1 levels were found at 1 hour after injury compared with control, and they either remained at similar levels or decreased to uninjured control levels by 3 hours after injury (Figure 5, A and B). FJ-B staining in adjacent sections to those used in the MALDI analysis revealed increases in the total number of FJ-B-positive cells with time after injury $\left(R^{2}=0.94, P=0.0012\right)$ (Figure 5, C and D) compared with uninjured control, supporting the possibility that early increases in LPA precursors within the thalamus at 1 hour after injury might indicate early cellular stress and/or neural degeneration that occurs at, and subsequent to, 3 hours after injury. 


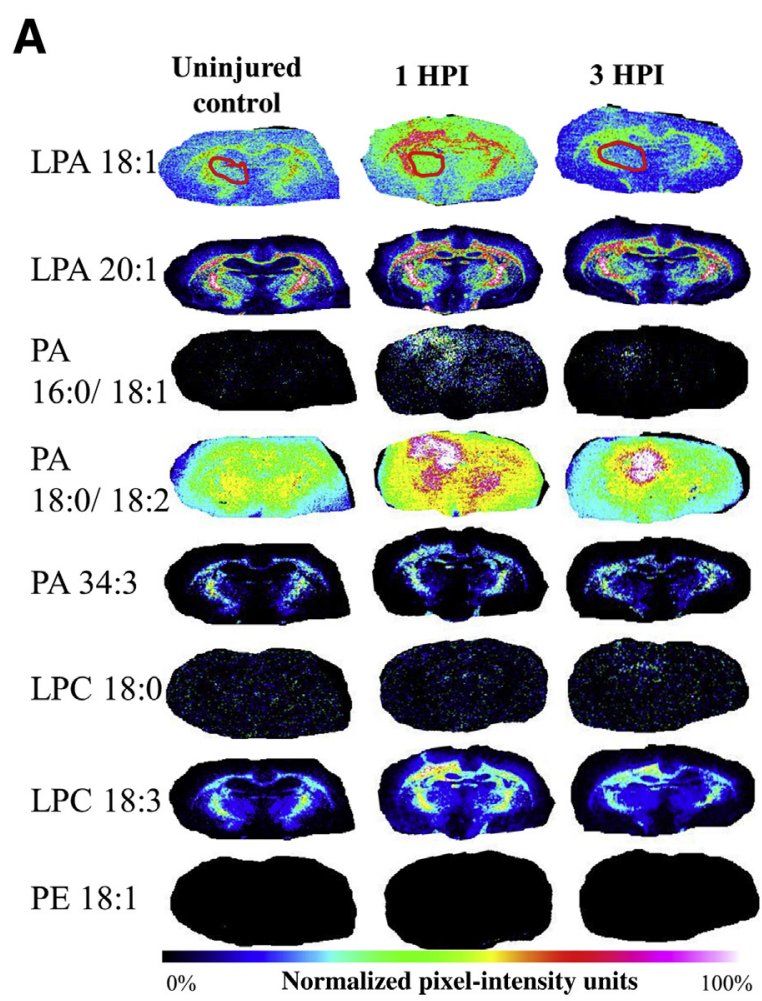

B

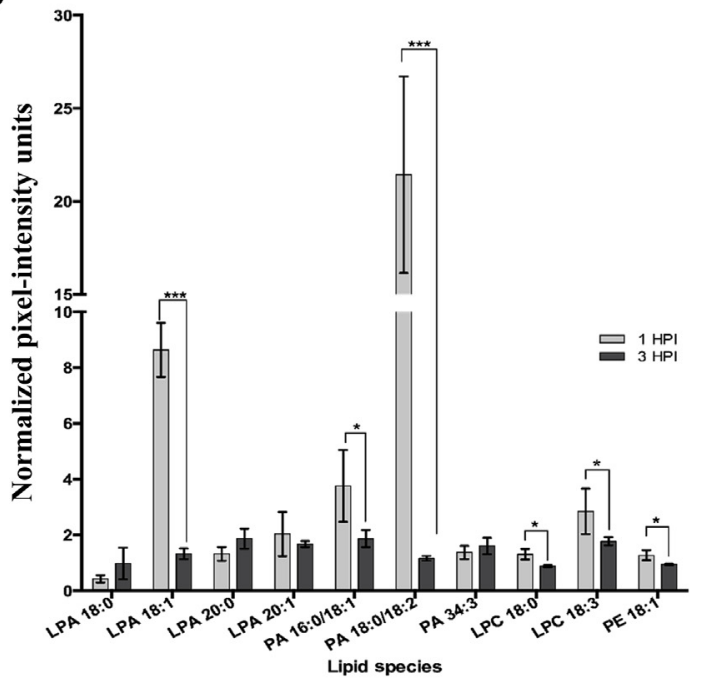

C

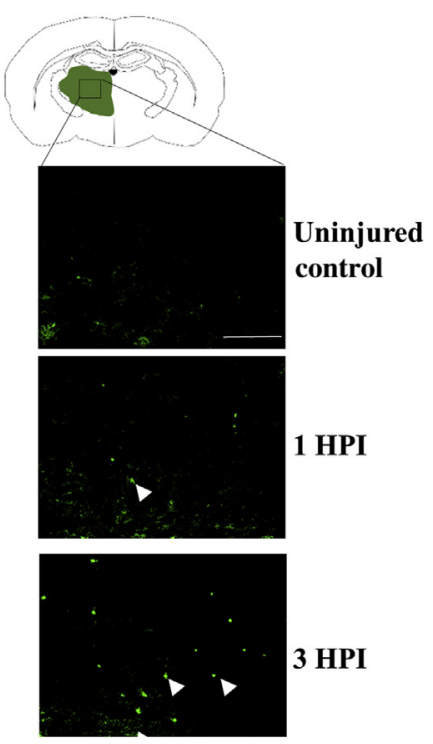

D

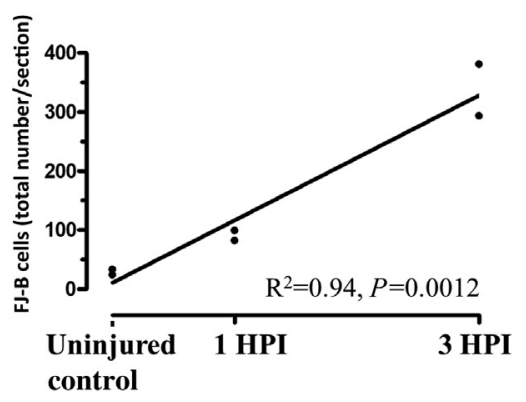

Figure 5 Lysophosphatidic acid (LPA) precursor expression precedes expression of neurodegeneration in the thalamus after injury. A: Mean ipsilateral thalamic regions of interest (ROIs; red circle) pixel intensity measurements of intracellular LPA precursors were acquired from representative matrix-assisted laser desorption ionization (MALDI) sections at bregma $-1.8 \mathrm{~mm}$ from all three groups. B: Normalized plot of thalamic ROI data at 1 and 3 hours after injury (HPI). C: Brain atlas at bregma $-1.8 \mathrm{~mm}$ showing the region where representative images were taken of necrotic/injured Fluoro-Jade B (FJ-B)-positive cells (examples shown with arrowheads) in sections adjacent to the MALDI images in uninjured controls, 1 hour after injury, and 3 hours after injury. D: Plot of total FJ-B-positive cell counts in thalamic regions (two rats/group) and the significant postinjury increase in number of degenerating cells. Data are expressed as means \pm SEM (B). $n=3$ per group (B). ${ }^{*} P<0.05,{ }^{* * *} P<0.001$ (two-tailed $t$-test, $95 \%$ CI in R). Scale bar $=100 \mu \mathrm{m}(\mathbf{C})$. LPC, lysophosphatidylcholine; $\mathrm{PA}$, phosphatidic acid; $\mathrm{PE}$, phosphatidylethanolamine.

\section{LPA Increases Are Regionally, but Not Globally, Associated with White Matter Damage}

Axons within the $\mathrm{CC}$ are particularly vulnerable to atrophy after contusion injury in the rodent because of the proximity to the primary impact zone, which results in axonal shearing as well as subsequent degeneration attributable to loss of cortical target cells. Altered lipid metabolism is likely to play a part in axonal degeneration because alterations in LPA and LPC have been shown to result in demyelination and degeneration of axons in the CNS. ${ }^{39-41}$ To investigate a potential role of LPA in axonal degeneration in the $\mathrm{CC}$ after 
A

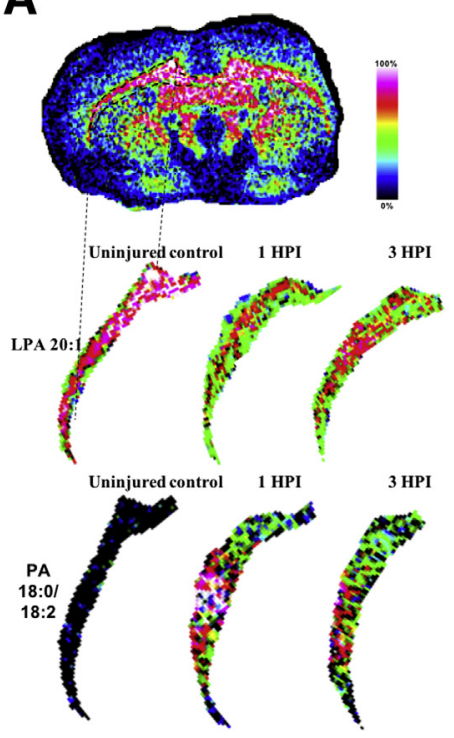

B

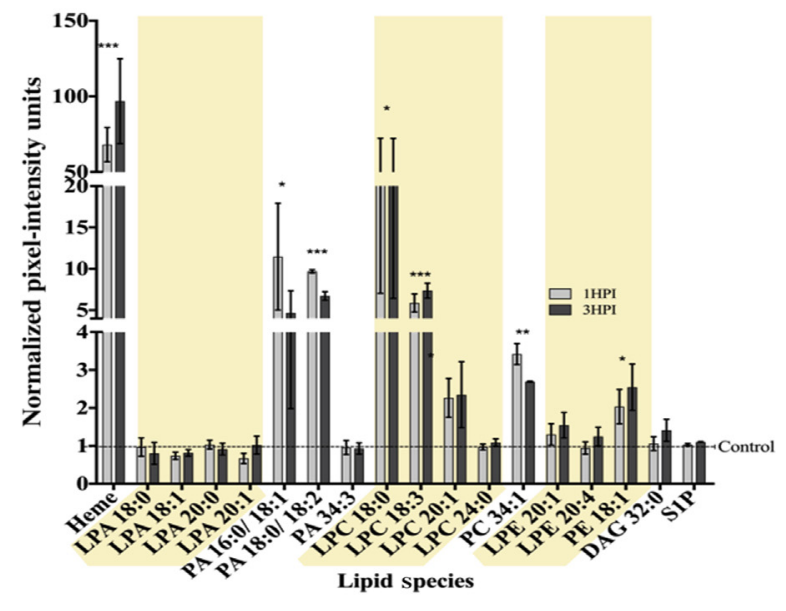

C
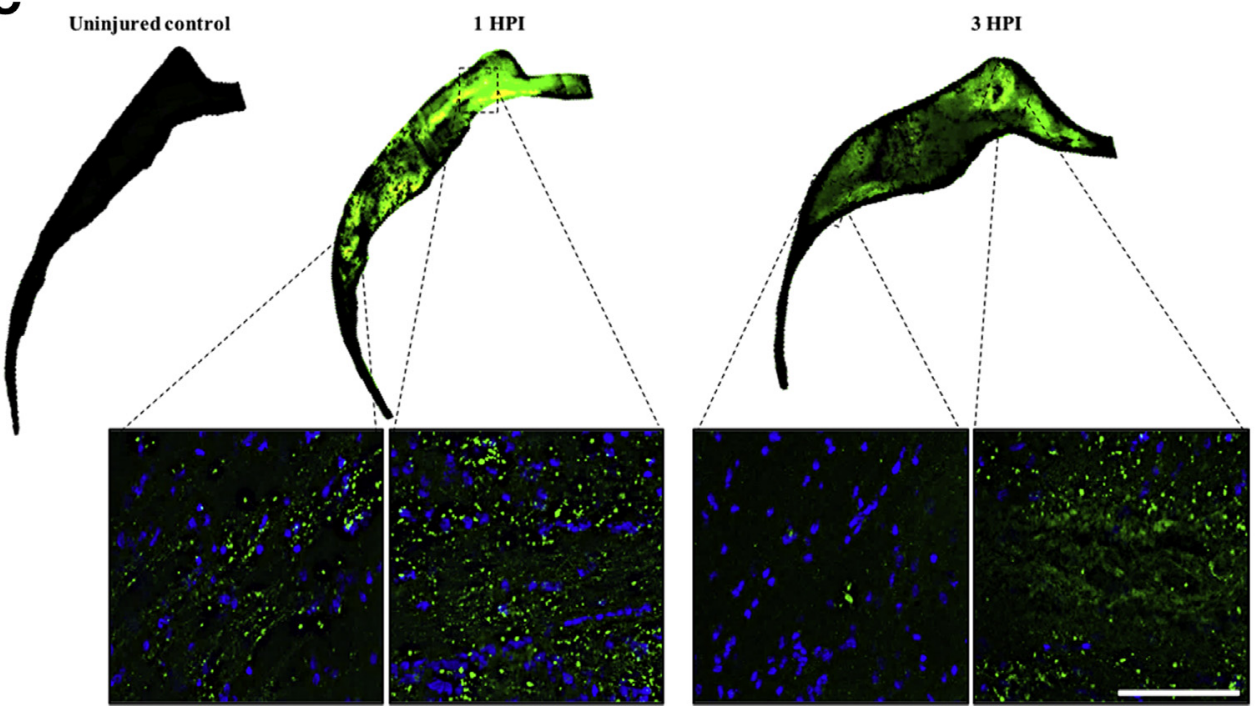

Figure 6 Lysophosphatidic acid (LPA) metabolites and markers of axonal injury are significantly increased in ipsilateral white matter after traumatic brain injury. A and B: Pixel intensity measurements taken for ipsilateral corpus callosum (marked with a dashed outline) for LPA 20:1 and phosphatidic acid (PA) 18:0/18:2 from uninjured control and injured brains at 1 and 3 hours after injury (HPI; $\mathbf{A}$ ) were selected to demonstrate the acute changes in lipid metabolites within the region and plotted graphically together with all lipid species identified from matrix-assisted laser desorption ionization (MALDI) in the white matter normalized to uninjured control pixel intensity values (B). Shaded regions highlight the 1-acyl (lyso-) phospholipid species. C: Immunostaining for the axonal injury marker $\beta A P P$ in adjacent sections from the same brains used for MALDI data acquisition (dashed outline in $\mathbf{A}$ ), and insets reveal accumulation of $\beta A P P$ (green) in similar regions to lipid increases; DAPI stain in blue. Data are expressed as means \pm SEM (B). $n=3$ per group $(\mathbf{B}) .{ }^{*} P<0.05,{ }^{* *} P<0.01$, and ${ }^{* *} P<0.001$ for the difference between 1 and 3 hours after injury (two-tailed $t$-test, $95 \%$ CI in R). Scale bar $=50 \mu \mathrm{m}$ (C). DAG, diacylglycerol; LPC, lysophosphatidylcholine; LPE, lysophosphatidylethanolamine; PC, phosphatidylcholine; PE, phosphatidylethanolamine; S1P, sphingosine-1-phosphate.

TBI, LPA expression was analyzed from ipsilateral ROIs of MALDI images over the entire $\mathrm{CC}$ at the epicenter of the injury ( $-1.34 \mathrm{~mm}$ to bregma) at 1 and 3 hours after injury. No significant injury-related changes were found in LPA species within the white matter compared with uninjured control levels (Figure 6, A and B), despite obvious increases in LPA within the entire white matter region at the injury epicenter (Figure 6, A and B). However, similar to other gray matter regions shown previously, the LPA precursors
(PA 16:0/18:1, LPC 18.0/18.3/20.1, phosphatidylcholine 34.1 , and PE 18.1) were all significantly increased within white matter by 2 to 20 NIUs above control values at both times after injury (Figure 6, A and B; Table 1). IHC for the axonal injury marker $\beta$ APP in adjacent tissue sections of the same animals used for MALDI IMS analysis showed increased APP staining at both times after injury (Figure 6C). This occurred in regions that appeared similar to the regional distribution of lipids on adjacent sections 

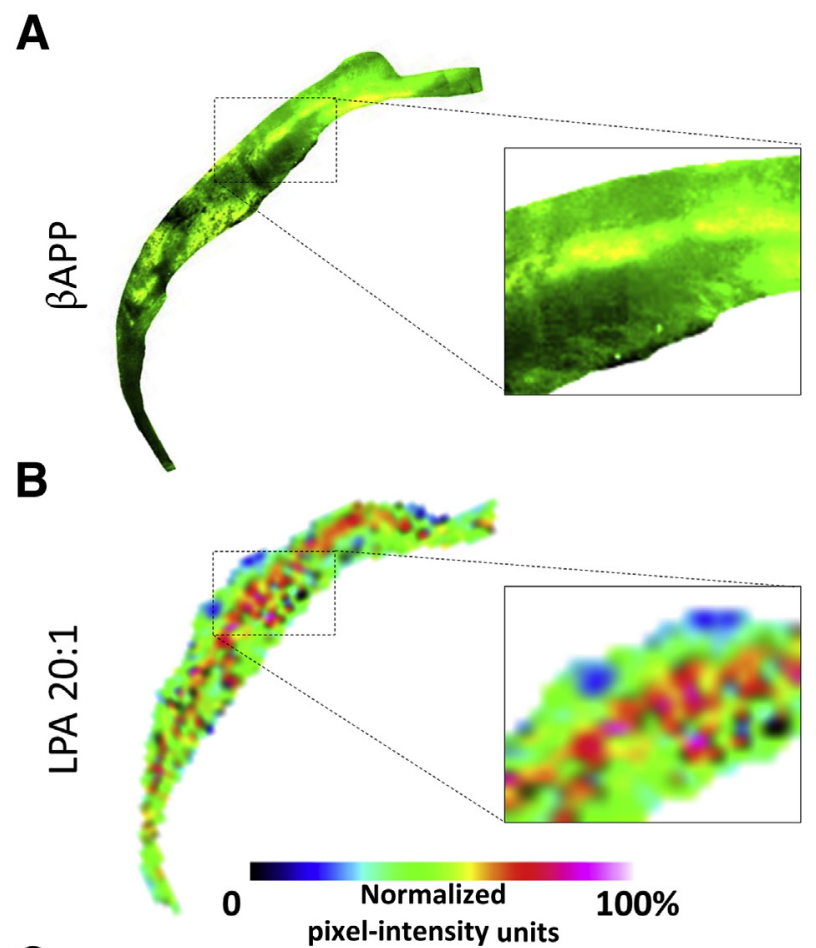

C

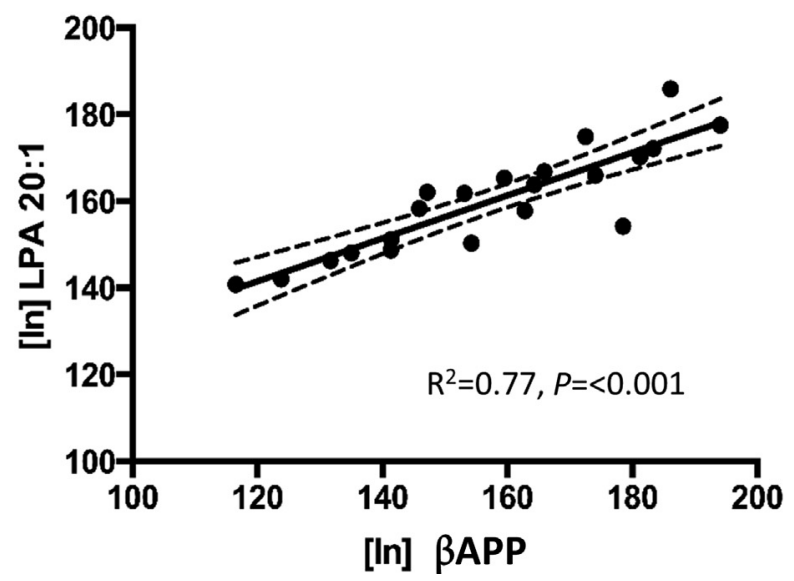

Figure 7 Spatial distribution of axonal injury marker $\beta$-amyloid precursor protein ( $\beta$ APP) correlates with specific lysophosphatidic acid (LPA) species and precursors. A and B: Pixel intensity images from a $400-\mu \mathrm{m}^{2}$ (21-pixel) region of corpus callosum from one brain at 1 hour after injury for BAPP (A) and LPA 20:1 (B). Boxed areas are shown at highter magnification in the insets. C: Image voxel-wise intensity correlation plots show that LPA positively correlates to $\beta$ APP signal $(P<0.001) . n=1$ per group (C). Original magnification, $\times 10$ (insets).

(Figure 6, A and C). Closer inspection of MALDI images revealed that, although LPA was globally enhanced in the $\mathrm{CC}$ after injury, there were distinct regional enhancements in LPA and PA expression at 1 and 3 hours after injury that may have been missed by the large white matter ROI used for analysis (Figure 6A). To more directly analyze spatial lipid changes in relation to axonal damage, 400 square pixel intensity maps, centered at the region of $\beta$ APP IHC enhancement, were aligned and overlaid onto all MALDI lipid images (including heme) in the same region on adjacent tissue sections in one injured brain at 1 hour after injury to test for any spatial correlation. Voxel-wise signal intensity correlation analysis showed that, unlike all other lipid species or lipid metabolites analyzed, LPA 20:1 signal intensity was the only lipid that correlated with the spatial distribution of $\beta$ APP image pixel intensity $(r=0.699$, $P<0.01$ ) (Figure 7, A-C); this result indicates that LPA expression might be spatially associated with axonal degeneration after TBI.

\section{Discussion}

\section{LPA and Disease Impact}

LPA is a promising therapeutic target for CNS injury. ${ }^{5}$ LPA is up-regulated in the CSF of both TBI mice and humans, consistent with work demonstrating the up-regulation of LPA receptors in the human brain after CNS neurotrauma. ${ }^{42}$ However, the source of the dysregulated LPA and the direct impact of LPA metabolism on the temporal and spatial progression of cellular pathologies in the injured adult brain remained relatively unexplored. This study is the first to identify LPA in the brain parenchyma and to find spatiotemporal correlations between LPA metabolism and cell death, as well as axonal injury and hemorrhaging throughout the brain after TBI using high-resolution MALDI IMS imaging and IHC.

\section{Enhanced LPA Metabolism as a Potential Marker of Diaschisis after TBI}

Although it is generally agreed that the entire brain responds to focal cortical injury, ${ }^{43,44}$ widespread changes in LPA within the intact brain have not been demonstrated after contusion injury, possibly because of limitations in technical resolution. Using high-resolution MALDI IMS enables a spatial representation of the early changes in LPA expression in the brain after injury could be assessed. The resulting MALDI images, therefore, provide a multilipid spatially unbiased readout of lipid distributions compared with more traditional biochemical analysis of isolated brain regions. Although recent publications have shown the utility of MALDI IMS in assessing changes in lipid distributions in the brain after blast injury ${ }^{45}$ and pericontusional changes after $\mathrm{CCI},{ }^{46}$ the current study provides early postinjury evidence of altered changes in specific lipids related to LPA and LPA metabolites in both proximal and remote regions of the brain after injury. The most pronounced expression occurred in white matter tracts throughout the brain, including the corpus callosum and the cerebellum. Several studies have demonstrated cerebellar injury associated with diaschisis after TBI, as characterized by changes in cellular metabolism in brain regions indirectly connected to the site of injury, and associated with long-term functional impairments. ${ }^{43,44,47,48}$ Prior metabolic studies have demonstrated that enhanced 
LPA signaling results in cell death in a variety of neural cell types. ${ }^{49-54}$ Given the association of LPA and metabolites with cell death observed in the current study, it is plausible, therefore, that the diaschisis-like pattern of LPA expression observed throughout the brain indicates underlying cellular pathology. Given the well-known excitotoxic action of LPA, ${ }^{11,52,55-57}$ these findings, therefore, suggest that the increase in LPA metabolism throughout the brain within 3 hours of injury may contribute to widespread damage not fully appreciated using other sampling methods.

\section{Injury Enhances Extracellular and Intracellular Expression of LPA Associated with Degeneration in the Pericontusion Cortex and Thalamus}

Recent TBI studies have shown increased LPA activity in the blood and CSF after injury ${ }^{5,42}$ and suggested that LPCautotaxin enzymatic activity in blood is the primary source of elevated LPA expression and subsequently LPA signaling through transmembrane G-protein-coupled receptor activation in the injured brain. ${ }^{20,42,58-61}$ However, the downstream functional consequences of LPA production in the adult brain from intracellular or extracellular stores remains controversial. ${ }^{62}$ The results from MALDI IMS analysis in this study indicate that pericontusional hemorrhagic gray matter regions are associated with the major increases in LPA and LPA precursor distribution after brain injury. However, alterations in LPA metabolism and enhanced intracellular LPA expression in nonheme regions were also observed, implying that changes in LPA metabolism are not confined to areas contaminated with blood, and that nonhemorrhagic effects of injury alone result in altered expression of intracellular LPA levels.

Within the pericontusional gray matter at 3 hours of injury, enhanced intracellular LPA expression was associated with neuronal degeneration; therefore changes in LPA signaling after TBI outside of hemorrhagic zones may also underlie neuronal death. The intracellular metabolic sources or receptors may contribute toward LPA-mediated cellular damage.

One of the early indicators of cortical injury in distal regions of the brain is thalamocortical atrophy. MALDI IMS analysis of the thalamus indicated a significant increase in the distribution of the intracellular precursor to LPA, PA 18:0/18:1, at 1 hour after injury. This increase was followed by an increase in neural degeneration at 3 hours after injury. The temporal and spatial changes in PA expression and neuronal degeneration tentatively suggest that aberrant PA expression may initiate the neurodegenerative signaling cascades within the thalamocortical pathway. PA expression is indirectly associated with neuronal degeneration within LPA and LPC signaling pathways, ${ }^{63,64}$ and therefore a plausible mechanism by which enhanced PA initiates thalamocortical atrophy may occur with enhanced intracellular LPA expression at subsequent postinjury times.
Bioactive Potent Phospholipid Species Enhanced in the Injured Brain

LPA alone is known to be a potent neuromodulator, ${ }^{65}$ and the relative potency of LPA's bioactivity is dependent on the species. Unsaturated species are more biologically potent than saturated species; in studies of smooth muscle cells, only unsaturated, and not saturated, LPA species resulted in increased proliferation and dedifferentiation. ${ }^{66-69}$ It is also known that increased synthesis of unsaturated LPA species 18:1 results in excitotoxicity as well as functional changes in neuronal cell types by activation of LPA receptors 1 and 2, and that this effect is self-propagating, triggering the production of more unsaturated LPA from intracellular stores ${ }^{7,70,71}$ and ultimately widespread cell death. ${ }^{72,73}$ Despite these data, no study has described the differential role of LPA species-specific changes in the pathogenesis of TBI in the adult brain. The results of the current study indicate enhanced metabolism of bioactive LPA isoforms 18:1 and 20:1 in the injured cortical gray matter, whereas the levels of saturated species did not change. Enhanced expression of unsaturated LPA likely originates from blood coagulation at the injury epicenter, and because unsaturated LPA has self-propagating effects, ${ }^{56}$ signaling of LPA will likely act as a catalyst for excitotoxic cellular responses in the injured brain. More chronic studies of LPA species changes are needed to thoroughly identify the differential effects of LPA saturation on cellular responses after injury. However, the current findings provide strong evidence that unsaturated LPA species are selectively involved in the early responses of the brain to injury and possibly the progression of TBI pathophysiology.

\section{Distribution of Phospholipids in the White Matter Is Associated with Axonal Injury}

Cortical injury in the adult brain cues the demyelination process within hours after injury, and this is observed in the corpus callosum as well as distal white matter regions. ${ }^{74-76}$ Although the molecules initiating the demyelination cascade after injury have yet to be identified, LPA receptor signaling is known to be a potent mediator of demyelination, ${ }^{39}$ and acute exogenous application of LPA induces demyelination of dorsal root neurons in ex vivo cultures. ${ }^{77}$ A recent study has also demonstrated LPA receptor type 1-mediated attenuation of axonal sprouting and regeneration after spinal cord injury. ${ }^{78}$ LPC also induces demyelination, at a slower rate in comparison to LPA, and is used for in vivo and in vitro models of multiple sclerosis. ${ }^{39,41}$ The temporal change in lipids in the ipsilateral corpus callosum shows a robust increase in LPC expression after injury in the present study and is in agreement with MALDI studies of ischemia, ${ }^{79,80}$ suggesting that after TBI, LPC synthesis may be indicative of global white matter injury. Furthermore, it is also possible that early LPC production precedes 
much greater LPA levels in the white matter through autotaxin activity.

PA, PE, and phosphatidylcholine are precursors to many phospholipid species, and their enhanced expression may be indicative of impending phospholipid metabolism, membrane hydrolysis, acylation of LPA or LPC, or diacylglycerol phosphorylation. ${ }^{81,82}$ The corpus callosum analysis revealed that, although there were no global white matter changes in LPA levels, there was a positive correlation between LPA 20:1 to regions of axonal injury, indicating that LPA may be a marker of axonal damage after TBI. Despite the robust data shown, further approaches, ideally using liquid chromatography-mass spectrometry, are required to assess the specificity and sensitivity of the MALDI data. In this way, larger group sizes can be more feasibly accommodated.

\section{Conclusion}

These data provide evidence of LPA as an early signaling molecule that contributes to the pathophysiology of TBI, not only within hemorrhagic regions, but widespread within the brain, and especially in white matter regions. The findings suggest that intracellular LPA signaling may play a role in the degeneration process after cortical trauma.

\section{Supplemental Data}

Supplemental material for this article can be found at https://doi.org/10.1016/j.ajpath.2018.05.005.

\section{References}

1. Saatman KE, Creed J, Raghupathi R: Calpain as a therapeutic target in traumatic brain injury. Neurotherapeutics 2010, 7:31-42

2. Ostrowski RP, Colohan AR, Zhang JH: Molecular mechanisms of early brain injury after subarachnoid hemorrhage. Neurol Res 2006, 28:399-414

3. Hasegawa Y, Suzuki H, Sozen T, Altay O, Zhang JH: Apoptotic mechanisms for neuronal cells in early brain injury after subarachnoid hemorrhage. Acta Neurochir Suppl 2011, 110:43-48

4. Weber JT: Calcium homeostasis following traumatic neuronal injury. Curr Neurovasc Res 2004, 1:151-171

5. Crack PJ, Zhang M, Morganti-Kossmann MC, Morris AJ, Wojciak JM, Fleming JK, Karve I, Wright D, Sashindranath M, Goldshmit Y, Conquest A, Daglas M, Johnston LA, Medcalf RL, Sabbadini RA, Pébay A: Anti-lysophosphatidic acid antibodies improve traumatic brain injury outcomes. J Neuroinflammation 2014, $11: 37$

6. Goldshmit Y, Matteo R, Sztal T, Ellett F, Frisca F, Moreno K, Crombie D, Lieschke GJ, Currie PD, Sabbadini RA, Pébay A: Blockage of lysophosphatidic acid signaling improves spinal cord injury outcomes. Am J Pathol 2012, 181:978-992

7. Goldshmit Y, Munro K, Leong SY, Pébay A, Turnley AM: LPA receptor expression in the central nervous system in health and following injury. Cell Tissue Res 2010, 341:23-32

8. Fernandis AZ, Wenk MR: Membrane lipids as signaling molecules. Curr Opin Lipidol 2007, 18:121-128
9. Adibhatla RM, Hatcher JF: Altered lipid metabolism in brain injury and disorders. Subcell Biochem 2008, 49:241-268

10. Underwood KW, Song C, Kriz RW, Chang XJ, Knopf JL, Lin L-L: A novel calcium-independent phospholipase A2, cPLA2-, that is prenylated and contains homology to cPLA2. J Biol Chem 1998, 273:21926-21932

11. Holtsberg FW, Steiner MR, Furukawa K, Keller JN, Mattson MP, Steiner SM: Lysophosphatidic acid induces a sustained elevation of neuronal intracellular calcium. J Neurochem 1997, 69:68-75

12. Möller T, Contos JJ, Musante DB, Chun J, Ransom BR: Expression and function of lysophosphatidic acid receptors in cultured rodent microglial cells. J Biol Chem 2001, 276:25946-25952

13. Shano S, Moriyama R, Chun J, Fukushima N: Lysophosphatidic acid stimulates astrocyte proliferation through LPA1. Neurochem Int 2008, 52:216-220

14. Jalink K, Eichholtz T, Postma FR, van Corven EJ, Moolenaar WH: Lysophosphatidic acid induces neuronal shape changes via a novel, receptor-mediated signaling pathway: similarity to thrombin action. Cell Growth Differ 1993, 4:247-255

15. Tigyi G, Fischer DJ, Sebök Á, Yang C, Dyer DL, Miledi R: Lysophosphatidic acid-induced neurite retraction in PC12 cells: control by phosphoinositide-Ca2+ signaling and Rho. J Neurochem 1996, 66: 537-548

16. Kadl A, Bochkov VN, Huber J, Leitinger N: Apoptotic cells as sources for biologically active oxidized phospholipids. Antioxid Redox Signal 2004, 6:311-320

17. Pasvogel AE, Miketova P, Moore IM: Differences in CSF phospholipid concentration by traumatic brain injury outcome. Biol Res Nurs 2010, 11:325-331

18. Santos-Nogueira E, Lopez-Serrano C, Hernandez J, Lago N, Astudillo AM, Balsinde J, Estivill-Torrus G, de Fonseca FR, Chun J, Lopez-Vales R: Activation of lysophosphatidic acid receptor type 1 contributes to pathophysiology of spinal cord injury. J Neurosci 2015, 35:10224-10235

19. Pagès C, Simon M-FF, Valet P, Saulnier-Blache JS: Lysophosphatidic acid synthesis and release. Prostaglandins Other Lipid Mediat 2001, 64:1-10

20. Aoki J, Inoue A, Okudaira S: Two pathways for lysophosphatidic acid production. Biochim Biophys Acta 2008, 1781:513-518

21. Gessel MM, Norris JL, Caprioli RM: MALDI imaging mass spectrometry: spatial molecular analysis to enable a new age of discovery. J Proteomics 2014, 107:71-82

22. Aichler M, Walch A: MALDI Imaging mass spectrometry: current frontiers and perspectives in pathology research and practice. Lab Invest 2015, 95:422-431

23. Spraggins JM, Rizzo DG, Moore JL, Rose KL, Hammer ND, Skaar EP, Caprioli RM: MALDI FTICR IMS of intact proteins: using mass accuracy to link protein images with proteomics data. J Am Soc Mass Spectrom 2015, 26:974-985

24. Yalcin EB, de la Monte SM: Review of matrix-assisted laser desorption ionization-imaging mass spectrometry for lipid biochemical histopathology. J Histochem Cytochem 2015, 63:762-771

25. Jones EE, Dworski S, Canals D, Casas J, Fabrias G, Schoenling D, Levade T, Denlinger C, Hannun YA, Medin JA, Drake RR: On-tissue localization of ceramides and other sphingolipids by MALDI mass spectrometry imaging. Anal Chem 2014, 86:8303-8311

26. Gessel M, Spraggins JM, Voziyan P, Hudson BG, Caprioli RM: Decellularization of intact tissue enables MALDI imaging mass spectrometry analysis of the extracellular matrix. J Mass Spectrom 2015, 50:1288-1293

27. Jenkinson M, Smith S: A global optimisation method for robust affine registration of brain images. Med Image Anal 2001, 5:143-156

28. Radeff-Huang J, Seasholtz TM, Matteo RG, Brown JH: G protein mediated signaling pathways in lysophospholipid induced cell proliferation and survival. J Cell Biochem 2004, 92:949-966

29. On NH, Savant S, Toews M, Miller DW: Rapid and reversible enhancement of blood-brain barrier permeability using lysophosphatidic acid. J Cereb Blood Flow Metab 2013, 33:1-11 
30. Tabuchi S, Kume K, Aihara M, Shimizu T: Expression of lysophosphatidic acid receptor in rat astrocytes: mitogenic effect and expression of neurotrophic genes. Neurochem Res 2000, 25: $573-582$

31. Eichholtz T, Jalink K, Fahrenfort I, Moolenaar WH: The bioactive phospholipid lysophosphatidic acid is released from activated platelets. Biochem J 1993, 291(Pt 3):677-680

32. Im DS, Heise CE, Harding MA, George SR, O'Dowd BF, Theodorescu D, Lynch KR: Molecular cloning and characterization of a lysophosphatidic acid receptor, Edg-7, expressed in prostate. Mol Pharmacol 2000, 57:753-759

33. Pagès C, Girard A, Jeanneton $\mathrm{O}$, Barbe $\mathrm{P}$, Wolf $\mathrm{C}$, Lafontan $\mathrm{M}$, Valet P, Saulnier-Blache JS: LPA as a paracrine mediator of adipocyte growth and function. Ann N Y Acad Sci 2000, 905: 159-164

34. McIntyre TM, Pontsler AV, Silva AR, St Hilaire A, Xu Y, Hinshaw JC, Zimmerman GA, Hama K, Aoki J, Arai H, Prestwich GD: Identification of an intracellular receptor for lysophosphatidic acid (LPA): LPA is a transcellular PPARgamma agonist. Proc Natl Acad Sci U S A 2003, 100:131-136

35. Thomsen GM, Le Belle JE, Harnisch JA, Mc Donald WS, Hovda DA, Sofroniew MV, Kornblum HI, Harris NG: Traumatic brain injury reveals novel cell lineage relationships within the subventricular zone. Stem Cell Res 2014, 13:48-60

36. Schmued LC, Hopkins KJ: Fluoro-Jade B: a high affinity fluorescent marker for the localization of neuronal degeneration. Brain Res 2000, 874:123-130

37. Kim J, Avants B, Patel S, Whyte J, Coslett BH, Pluta J, Detre JA, Gee JC: Structural consequences of diffuse traumatic brain injury: a large deformation tensor-based morphometry study. Neuroimage 2008, 39:1014-1026

38. Smith DH, Chen XH, Pierce JES, Wolf JA, Trojanowski JQ, Graham DI, Mcintosh TK: Progressive atrophy and neuron death for one year following brain trauma in the rat. J Neurotrauma 1997, 14: $715-727$

39. Nagai J, Uchida H, Matsushita Y, Yano R, Ueda M, Niwa M, Aoki J, Chun J, Ueda H: Autotaxin and lysophosphatidic acid1 receptormediated demyelination of dorsal root fibers by sciatic nerve injury and intrathecal lysophosphatidylcholine. Mol Pain 2010, 6:78

40. Inoue M, Xie W, Matsushita Y, Chun J, Aoki J, Ueda H: Lysophosphatidylcholine induces neuropathic pain through an action of autotaxin to generate lysophosphatidic acid. Neuroscience 2008, 152: 296-298

41. Vereyken EJF, Fluitsma DM, Bolijn MJ, Dijkstra CD, Teunissen CE: An in vitro model for de- and remyelination using lysophosphatidyl choline in rodent whole brain spheroid cultures. Glia 2009, 57: $1326-1340$

42. Frugier T, Crombie D, Conquest A, Tjhong F, Taylor C, Kulkarni T, McLean C, Pébay A: Modulation of LPA receptor expression in the human brain following neurotrauma. Cell Mol Neurobiol 2011, 31: 569-577

43. Hovda DA, Lee SM, Smith ML, Von Stuck S, Bergsneider M, Kelly D, Shalmon E, Martin N, Caron M, Mazziotta J: The neurochemical and metabolic cascade following brain injury: moving from animal models to man. J Neurotrauma 1995, 12:903-906

44. Nishibe M, Barbay S, Guggenmos D, Nudo RJ: Reorganization of motor cortex after controlled cortical impact in rats and implications for functional recovery. J Neurotrauma 2010, 27:2221-2232

45. Woods AS, Colsch B, Jackson SN, Post J, Baldwin K, Roux A, Hoffer B, Cox BM, Hoffer M, Rubovitch V, Pick CG, Schultz JA, Balaban C: Gangliosides and ceramides change in a mouse model of blast induced traumatic brain injury. ACS Chem Neurosci 2013, 4: 594-600

46. Roux A, Muller L, Jackson SN, Post J, Baldwin K, Hoffer B, Balaban CD, Barbacci D, Schultz JA, Gouty S, Cox BM, Woods AS: Mass spectrometry imaging of rat brain lipid profile changes over time following traumatic brain injury. J Neurosci Methods 2016, 272: $19-32$

47. Stein DG, Hoffman SW: Concepts of CNS plasticity in the context of brain damage and repair. J Head Trauma Rehabil 2003, 18:317-341

48. Holschneider DP, Guo Y, Wang Z, Roch M, Scremin OU: Remote brain network changes after unilateral cortical impact injury and their modulation by acetylcholinesterase inhibition. J Neurotrauma 2013, 30:907-919

49. Frisca F, Crombie DE, Dottori M, Goldshmit Y, Pébay A: Rho/ROCK pathway is essential to the expansion, differentiation, and morphological rearrangements of human neural stem/progenitor cells induced by lysophosphatidic acid. J Lipid Res 2013, 54:1192-1206

50. Furui T, LaPushin R, Mao M, Khan H, Watt SR, Watt MAV, Lu Y, Fang X, Tsutsui S, Siddik ZH, Bast RC, Mills GB: Overexpression of Edg-2/vzg-1 induces apoptosis and anoikis in ovarian cancer cells in a lysophosphatidic acid-independent manner. Clin Cancer Res 1999, 5: 4308-4318

51. Goetzl EJ, Kong Y, Mei B: Lysophosphatidic acid and sphingosine 1phosphate protection of $\mathrm{T}$ cells from apoptosis in association with suppression of Bax. J Immunol 1999, 162:2049-2056

52. Holtsberg FW, Steiner MR, Keller JN, Mark RJ, Mattson MP, Steiner SM: Lysophosphatidic acid induces necrosis and apoptosis in hippocampal neurons. J Neurochem 1998, 70:66-76

53. Lai J-M, Lu C-Y, Yang-Yen H-F, Chang Z-F: Lysophosphatidic acid promotes phorbol-ester-induced apoptosis in TF-1 cells by interfering with adhesion. Biochem J 2001, 359(Pt 1):227-233

54. Ye X, Ishii I, Kingsbury MA, Chun J: Lysophosphatidic acid as a novel cell survival/apoptotic factor. Biochim Biophys Acta 2002, 1585:108-113

55. Steiner MR, Urso JR, Klein J, Steiner SM: Multiple astrocyte responses to lysophosphatidic acids. Biochim Biophys Acta 2002, 1582:154-160

56. Ma L, Nagai J, Chun J, Ueda H: An LPA species (18:1 LPA) plays key roles in the self-amplification of spinal LPA production in the peripheral neuropathic pain model. Mol Pain 2013, 9:29

57. Hildebrandt JP, Hildebrandt P: Lysophosphatidic acid depletes intracellular calcium stores different from those mediating capacitative calcium entry in C6 rat glioma cells. Glia 1997, 19: $67-73$

58. Gierse J, Thorarensen A, Beltey K, Bradshaw-Pierce E, CortesBurgos L, Hall T, Johnston A, Murphy M, Nemirovskiy O, Ogawa S, Pegg L, Pelc M, Prinsen M, Schnute M, Wendling J, Wene S, Weinberg R, Wittwer A, Zweifel B, Masferrer J: A novel autotaxin inhibitor reduces lysophosphatidic acid levels in plasma and the site of inflammation. J Pharmacol Exp Ther 2010, 334: $310-317$

59. Bolen AL, Naren AP, Yarlagadda S, Beranova-Giorgianni S, Chen L, Norman D, Baker DL, Rowland MM, Best MD, Sano T, Tsukahara T, Liliom K, Igarashi Y, Tigyi G: The phospholipase A1 activity of lysophospholipase A-I links platelet activation to LPA production during blood coagulation. J Lipid Res 2011, 52: 958-970

60. Moolenaar WH, van Meeteren LA, Giepmans BNG: The ins and outs of lysophosphatidic acid signaling. Bioessays 2004, 26:870-881

61. Ferry G, Tellier E, Try A, Grés S, Naime I, Simon MF, Rodriguez M, Boucher J, Tack I, Gesta S, Chomarat P, Dieu M, Raes M, Galizzi JP, Valet P, Boutin JA, Saulnier-Blache JS: Autotaxin is released from adipocytes, catalyzes lysophosphatidic acid synthesis, and activates preadipocyte proliferation: upregulated expression with adipocyte differentiation and obesity. J Biol Chem 2003, 278:18162-18169

62. Mills GB, Moolenaar WH: The emerging role of lysophosphatidic acid in cancer. Nat Rev Cancer 2003, 3:582-591

63. Adibhatla RM, Hatcher JF: Role of lipids in brain injury and diseases. Future Lipidol 2007, 2:403-422 
64. Titsworth W, Liu N-K, Xu X-M: Role of secretory phospholipase A2 in CNS inflammation: implications in traumatic spinal cord injury. CNS Neurol Disord Drug Targets 2008, 7:254-269

65. Choi JW, Herr DR, Noguchi K, Yung YC, Lee C-W, Mutoh T, Lin M-E, Teo ST, Park KE, Mosley AN, Chun J: LPA receptors: subtypes and biological actions. Annu Rev Pharmacol Toxicol 2010, 50:157-186

66. Tokumura A, Iimori M, Nishioka Y, Kitahara M, Sakashita M, Tanaka S: Lysophosphatidic acids induce proliferation of cultured vascular smooth muscle cells from rat aorta. Am J Physiol 1994, 267:C204-C210

67. Jalink K, Hengeveld T, Mulder S, Postma FR, Simon MF, Chap H, Van Der Marel GA, Van Boom JH, Van Blitterswijk WJ, Moolenaar WH: Lysaphosphatidic acid-induced Ca2+ mobilization in human A431 cells: structure-activity analysis. Biochem J 1995, 307:609-616

68. Yoshida K: Vascular remodeling induced by naturally occurring unsaturated lysophosphatidic acid in vivo. Circulation 2003, 108: $1746-1752$

69. Hayashi K, Takahashi M, Nishida W, Yoshida K, Ohkawa Y, Kitabatake A, Aoki J, Arai H, Sobue K: Phenotypic modulation of vascular smooth muscle cells induced by unsaturated lysophosphatidic acids. Circ Res 2001, 89:251-258

70. Lee C-WW, Rivera R, Gardell S, Dubin AE, Chun J: GPR92 as a new G12/13- and Gq-coupled lysophosphatidic acid receptor that increases cAMP, LPA5. J Biol Chem 2006, 281:23589-23597

71. Yano R, Ma L, Nagai J, Ueda H: Interleukin- $1 \beta$ plays key roles in LPA-induced amplification of LPA production in neuropathic pain model. Cell Mol Neurobiol 2013, 33:1033-1041

72. Farooqui AA, Yang HC, Rosenberger TA, Horrocks LA: Phospholipase A2 and its role in brain tissue. J Neurochem 1997, 69:889-901
73. Kagan VE, Quinn PJ: Phospholipid Metabolism in Apoptosis. New York, NY: Springer, 2002

74. Xu S, Zhuo J, Racz J, Shi D, Roys S, Fiskum G, Gullapalli R: Early microstructural and metabolic changes following controlled cortical impact injury in rat: a magnetic resonance imaging and spectroscopy study. J Neurotrauma 2011, 28:2091-2102

75. Shi H, Hu X, Leak RK, Shi Y, An C, Suenaga J, Chen J, Gao Y: Demyelination as a rational therapeutic target for ischemic or traumatic brain injury. Exp Neurol 2015, 272:17-25

76. Liu MC, Akle V, Zheng W, Kitlen J, O'Steen B, Larner SF, Dave JR, Tortella FC, Hayes RL, Wang KKW: Extensive degradation of myelin basic protein isoforms by calpain following traumatic brain injury. J Neurochem 2006, 98:700-712

77. Inoue M, Rashid MH, Fujita R, Contos JJ, Chun J, Ueda H: Initiation of neuropathic pain requires lysophosphatidic acid receptor signaling. Nat Med 2004, 10:712-718

78. Fink KL, López-Giráldez F, Kim I-J, Strittmatter SM, Cafferty WBJ: Identification of intrinsic axon growth modulators for intact CNS neurons after injury. Cell Rep 2017, 18:2687-2701

79. Wang H-YJ, Wu H-W, Tsai P-J, Liu CB: MALDI-mass spectrometry imaging of desalted rat brain sections reveals ischemia-mediated changes of lipids. Anal Bioanal Chem 2012, 404:113-124

80. Shanta SR, Zhou L-H, Park YS, Kim YH, Kim Y, Kim KP: Binary matrix for MALDI imaging mass spectrometry of phospholipids in both ion modes. Anal Chem 2011, 83:1252-1259

81. Anliker B, Chun J: Cell surface receptors in lysophospholipid signaling. Semin Cell Dev Biol 2004, 15:457-465

82. Birgbauer E, Chun J: New developments in the biological functions of lysophospholipids. Cell Mol Life Sci 2006, 63:2695-2701 